\title{
Picamalácia na Gestação de Risco e Aspectos Psicológicos Relacionados
}

\author{
Ana Cristina Barros da Cunha ${ }^{1}$ \\ Departamento de Psicologia Clínica, Instituto de Psicologia, Universidade Federal \\ do Rio de Janeiro, Rio de Janeiro, RJ, Brazil \\ Programa de Mestrado Profissional em Saúde Perinatal da Maternidade Escola \\ da Universidade Federal do Rio de Janeiro, Rio de Janeiro, RJ, Brasil \\ Programa de Pós-Graduação em Psicologia, Universidade Federal do Espírito Santo, \\ Vitória, ES, Brazil \\ Solange Frid Patricio \\ Maternidade Escola da Universidade Federal do Rio de Janeiro, Rio de Janeiro, RJ, Brazil \\ Programa de Pós-Graduação em Saúde da Criança e da Mulher, Instituto Fernandes \\ Figueiras, Fundação Oswaldo Cruz, Rio de Janeiro, RJ, Brazil \\ Laila Pires Ferreira Akerman \\ Paula Sampaio Maynarde \\ Instituto de Psicologia, Universidade Federal do Rio de Janeiro, Rio de Janeiro, RJ, Brazil \\ Claudia Saunders \\ Programa de Mestrado Profissional em Saúde Perinatal da Maternidade Escola \\ da Universidade Federal do Rio de Janeiro, Rio de Janeiro, RJ, Brasil \\ Programa de Pós-Graduação em Nutrição, Instituto de Nutrição Josué de Castro, \\ Universidade Federal do Rio de Janeiro, Rio de Janeiro, RJ, Brazil
}

\section{Resumo}

Com base em delineamento descritivo e exploratório, objetivou-se avaliar indicadores emocionais (sinais de estresse, ansiedade e depressão) e de enfrentamento (coping) em sete gestantes adultas com Diabetes Mellitus e relato de práticas de picamalácia. A prática de picamalácia na gestação atual foi identificada por meio de entrevista validada, antes das participantes responderem aos instrumentos para avaliação psicológica: (a) Inventário de sinais e sintomas de stress de Lipp (ISSL), para avaliar estresse; (b) Escalas BECK, Inventário BAI e BDI, para avaliar ansiedade e depressão; (c) Escalas Modos de Enfrentamento de Problemas (EMEP), para identificas estratégias de enfrentamento frente ao estresse; e (d) Roteiro de entrevista elaborado para pesquisa. As gestantes foram entrevistadas individualmente e seu relato foi analisado em macro-categorias pela metodologia de Bardin. Indicadores de estresse, ansiedade e depressão foram encontrados em todas as gestantes e as práticas de picamalácia mais referidas foram pagofagia $(30,8 \%)$ e ingestão de fruta com sal $(30,8 \%)$. Todas as gestantes apresentavam em comum uma sensação de satisfação após a prática da picamalácia. Tendo em vista possíveis associações entre este transtorno alimentar e desfechos desfavoráveis, considera-se importante investigar a picamalacia a fim de propor uma assistência pré-natal mais efetiva na melhora da saúde da díade mãe-filho.

Palavras-chave: Gestação, diabetes mellitus, picamalácia, ansiedade, depressão, enfrentamento.

Endereço para correspondência: Rua das Laranjeiras, 180, Laranjeiras, Rio de Janeiro, RJ, Brasil 22240-003. E-mail: acbcunha@yahoo.com.br

Financiamento: Fundação de Amparo à Pesquisa do Estado do Rio de Janeiro (FAPERJ); Programa Institucional de Bolsas de Iniciação Científica (PIBIC)/ Universidade Federal do Rio de Janeiro (UFRJ). 


\title{
Pica in the Pregnancy and Psychological Aspects Related
}

\begin{abstract}
Based on a descriptive and exploratory research design, this study aimed to evaluate emotional (sings of stress, anxiety and depression) and coping indicators in seven pregnant women with diabetes and pica reporting practices. The pica reporting practices in their current pregnancies were identified before they answered the following instruments for psychological evaluation: (a) Inventory of signs and symptoms of stress of Lipp (ISSL), to evaluate stress; (b) BECK Scales, BAI and BDI Inventory, to evaluate anxiety and depression; (c) Scales of Ways of Coping (EMEP), to identify coping strategies to deal with stress; (d) an Interview Guide created for this study. The pregnant women were interviewed individually and their reports were analyzed into macro-categories by the Bardin's methodology. Indicators of stress, anxiety and depression were found in all pregnant women, whose most reported practice was pagofagia (30.8\%) and fruit with salt (30.8\%). The verbal report revealed that all women had a feeling of satisfaction after the practice of pica. Given that the associations between this eating disorder and unfavorable outcomes, it is important to investigate the pica in order to propose a more effective prenatal care in improving the health of the mother-child dyad.
\end{abstract}

Keywords: Pregnancy, diabetes mellitus, Pica, anxiety, depression, coping.

\section{Pica en el Embarazo y Aspectos Psicologicos Relacionados}

\section{Resumen}

Basado en un diseño descriptivo y exploratorio, el objetivo del estudio fue evaluar los indicadores de estrés, ansiedad, depresión y afrontamiento en siete mujeres embarazadas diagnosticadas con diabetes y informes de la pica. La práctica de pica fue identificada a través de una entrevista aplicada antes de los siguientes instrumentos de evaluación psicológica: (a) Inventario de los signos y síntomas de estrés Lipp (ISSL), para evaluar el estrés; (b) Escalas de BECK de Ansiedad y Depresión (BAI y BDI), para evaluar la ansiedad y la depresión; (c) Escala Modos de Afrontamiento de Problemas (EMEP), para evaluar las estrategias de afrontamiento delante el estrés y (d) Entrevista individual preparada para la investigación. Las entrevistas fueran analizadas por la metodología de Bardin. Indicadores de estrés, ansiedad y depresión se encontraron en todas ellas, la práctica de pica más reportada fue pagofagia $(30,8 \%)$ y las frutas con sal (30,8\%). Las participantes reportaron un sentido de satisfacción después de la práctica. Teniendo en cuenta las asociaciones entre los trastornos alimentarios y desenlaces desfavorables, se considera importante investigar la Pica con el fin de proponer un cuidado prenatal más eficaz para la mejora de la salud de la díada madre-hijo.

Palavras clave: Embarazo, diabetes mellitus, Pica, ansiedad, depresión, afrontamiento.

A gravidez é um momento de grandes expectativas e mudanças na vida da mulher, caracterizado por um estado emocional de insegurança, medos e angústias (Sarmento \& Setúbal, 2003). É um período ainda mais crítico quando ocorre sob condições clínicas de risco fetal e/ou materno, como, por exemplo, a Diabetes Mellitus Gestacional (DMG) ${ }^{2}$. Dentre os diferen-

2 O DMG é definido pela Sociedade Brasileira de tes tipos de gestação de risco, a gravidez com

Diabetes (SBD, 2015) como qualquer intolerância à glicose, de magnitude variável, com início ou diagnóstico na gestação. O DMG cursa com diminuição mais acentuada da sensibilidade à insulina e a gestante fica vulnerável à ocorrência de comprometimento fetal devido a hiperglicemia materna (macrossomia fetal, crescimento fetal incompatível com a idade gestacional), resultando em maior chance de ocorrência de complicações no parto e necessidade de parto cirúrgico, hipoglice- 
DMG resulta em estresse ${ }^{3}$ significativo, quando as gestantes são mais vulneráveis a problemas físicos (morte fetal, macrossomia) e psíquicos (estresse, ansiedade e depressão) durante a gravidez (Silva, Santos, \& Parada, 2004). Tais riscos podem provocar a vivencia de sofrimento psíquico na gestante devido ao significativo impacto desse diagnóstico (Araújo, Pessoa, Damasceno, \& Zanetti, 2013). Além disso, gestantes com DMG são mais propensas a apresentar sinais de depressão (Nicklas et al., 2013) e de ansiedade (Morrison, Lowe, \& Collins, 2014); além da probabilidade de desenvolver Diabetes Mellitus tipo 2 no pós-parto (Morrison et al., 2014; Nolan, McCrone, \& Chertok, 2011). Tudo isso resulta em condições psicoafetivas desfavoráveis ao desenvolvimento da díade mãe-bebê, com repercussões no vínculo afetivo entre a mulher, o bebê e sua família, particularmente característico em uma gravidez de risco (Quevedo, Lopes, \& Lefevre, 2006; Silva et al., 2004).

Muitas vezes para lidar com essa condição estressante, a mulher passa a adotar estratégias não adequadas e pouco resilientes para dar conta da instabilidade emocional que passam a vivenciar quando podem aparecer os transtornos alimentares na gravidez (Ayeta, Cunha, Hendelman, \& Saunders, 2015). De acordo com Dunker, Alvarenga e Alves (2009), esses transtornos estão associados às mudanças metabólicas, endócrinas, psicológicas e nutricionais, que geram efeitos negativos tanto para a mulher quanto para o feto, incluindo alta prevalência de abortos, baixo peso ao nascer, complicações obstétricas e depressão pós-parto (Dunker et al., 2009).

mia neonatal, distúrbios respiratórios e até morte fetal intrauterina (SBD, 2015; Sociedade Brasileira de Endocrinologia e Metabologia, 2008).

3 O estresse pode ser definido como toda reação do organismo, juntamente com os componentes psicológicos, físicos, mentais e hormonais que ocorrem frente à necessidade de uma grande adaptação a um evento estressor, mas que será prejudicial se houver exposição prolongada ao evento ou uma predisposição do indivíduo a determinadas doenças (Lipp, 2000, p. 109).
Nesse contexto, observa-se uma desordem alimentar chamada Picamalácia, conhecida como . . . a ingestão persistente de substâncias inadequadas com pequeno ou nenhum valor nutricional, ou de substâncias comestiveis, mas não na sua forma habitual (American Psychiatric Association, 2013; Ayeta et al., 2015; Saunders et al., 2009, p. 441), que tem sido pouco estudada, principalmente no que se refere aos aspectos psicológicos associados. O Diagnostic and Statistical Manual of Mental Disorders (DSM-V), da American Psychiatric Association (2013), define como critérios de classificação para o diagnóstico da Picamalácia o seguinte: (a) ingestão persistente de substâncias não nutritivas por um período de pelo menos 1 mês; (b) ingestão de substâncias não nutritivas inapropriada para o nível de desenvolvimento; (c) comportamento alimentar que não faz parte da cultura praticada; (d) comportamento alimentar que ocorre exclusivamente durante o curso de um transtorno mental. Sendo assim, o diagnóstico dessa desordem só poderá ser feito no caso de severidade da frequência do comportamento alimentar que legitime uma indicação clínica independente (American Psychiatric Association, 2013).

Raros estudos apontam essa desordem como associada a uma prática na gestação culturalmente aceita em diferentes contextos histórico-culturais (Kachani \& Cordás, 2009; Thihalolipavan, Candalla, \& Ehrlich, 2013; Young, 2010), podendo ocorrer, inclusive, entre gestantes de classes sociais mais privilegiadas (Mikkelsen, Andersen, \& Olsen, 2006).

Literatura tem relacionado a prática da picamalácia com a compensação de níveis baixos de ferro e/ou alívio da sintomatologia especifica da gravidez, como enjoos, náuseas e edemas, sendo transmitida no contexto familiar como uma crença transgeracional (Ahmed, Abdullahi, \& Adam, 2012; Kachani \& Cordás, 2009).

Estudos sugerem que existem aspectos emocionais relevantes associados a essa desordem, que é mais frequentemente estudada em mulheres gestantes devido aos riscos para o bebê (Ahmed et al., 2012; Simpson, Mull, Longley, \& East, 2000; Thihalolipavan et al., 2013). As 
substâncias inadequadas consumidas, por vezes tóxicas como talco, cinzas de cigarro, giz, sabonete, podem se associar com elevação da pressão arterial, ultrapassar a barreira placentária e atingir o feto, ocasionando prematuridade, mortalidade perinatal, baixo peso, diminuição do perímetro encefálico e até aborto (Thihalolipavan et al., 2013). A longo prazo, inclusive, a picamalácia pode ocasionar no desenvolvimento infantil comprometimentos cognitivos e de comunicação (Erdem, Hernandez, Kyono, Chan-Nishina, \& Iwaishi, 2004).

Devido aos graves desfechos obstétricos resultantes dessa prática durante a gravidez, o diagnóstico e tratamento imediato dessa desordem são fundamentais, ainda mais se considerando que é um transtorno cuja causa e tratamento são ainda discutíveis (Bhatia \& Kaur, 2014; Fiestas-Teque, 2014). Nos poucos estudos existentes é consenso de que a picamalácia está associada ao estresse durante a gravidez, principalmente ao aumento do estresse materno no $1^{\circ}$ trimestre gestacional, quando existe maior rigor no controle da alimentação, o que causaria ansiedade associada à irritabilidade, com consequente ingestão de alimentos não nutritivos (picamalácia) como forma de alívio da ansiedade e do estresse (Kachani \& Cordás, 2009; Young, 2010). Nessa perspectiva, Young (2010) afirma que a picamalácia pode ser considerada como uma resposta de proteção do indivíduo ao estresse psicológico, ou seja, um "mediador do estresse" que atuaria no sistema imunológico. Entretanto, essa hipótese é ambígua e o próprio autor julga necessário realizar novos estudos com maior rigor metodológico e de caráter longitudinal, que permitam confirmar tal hipótese e esclarecer a relação entre picamalácia e gravidez (Young, 2010).

Nessa perspectiva, ainda que a etiologia da picamalácia seja complexa e multifatorial, tem sido sugerida possível relação entre fatores emocionais e a prática da picamalácia. Discutem-se associações entre alterações hormonais e estresse (Simpson et al., 2000), já que na gravidez um crescente aumento na síntese de hormônios femininos pode causar alterações no organismo materno para o crescimento e desenvolvimento fetal adequado (Vieira \& Parizotto, 2013).
Dessa forma, tais alterações hormonais podem ocasionar mudanças físicas e psicológicas significativas na mulher, que resultariam em sintomatologia depressiva, ansiedade, baixa concentração, irritabilidade, mudança no apetite, insônia, hipersônia e perda de energia (Simpson et al., 2000; Vieira \& Parizoto, 2013).

Algumas dessas condições emocionais, como a ansiedade, por exemplo, acompanham todo o ciclo grávido-puerperal, gerando, por vezes, estados de insatisfação, intranquilidade, insegurança, incerteza e medos, que, como dito anteriormente, atualiza as angústias e ansiedades características das mudanças que ocorrem ao longo de toda gestação (Sarmento \& Setúbal, 2003). Dessa forma, esse estado emocional característico da gravidez pode resultar em estresse e afetar o processo de enfrentamento (coping ${ }^{4}$ ) da mulher gestante frente à vulnerabilidade típica desse período. Sob condições emocionas desfavoráveis, a gestante acaba por adotar estratégias de enfrentamento pouco funcionais ou protetoras do desenvolvimento da díade mãe-bebê, como é o caso da picamalácia. No entanto, tal hipótese precisa ser ainda investigada em contextos específicos de vulnerabilidade, como a gravidez de risco com DMG, uma vez que são recentes e raríssimos os estudos que discutem a relação entre gravidez com DMG e picamalácia, sobretudo estudos nacionais (Ayeta et al., 2015). Estes poucos estudos se referem a uma possível associação entre gestação com DMG e a prática da picamalácia, considerando que a diabetes na gestação implica em maior chance de desenvolver este transtorno (Ayeta et al., 2015; Ezzeddin, Zavoshy, Noroozi, Jahanihashemi, \& Riseh, 2015; Gangopadhyay, Karoshi, \& Keith, 2011); porém, somente um destes estudos discute os aspectos psicológicos relacionados (Ayeta et al., 2015).

Cabe ainda ressaltar que, a picamalácia na gestação é uma desordem com difícil diagnós-

4 Considera-se a resposta de coping como uma ação intencional, física ou mental, iniciada em resposta a um estressor percebido, dirigida para circunstâncias externas ou estados internos (medo, ansiedade, angústia; Lazarus \& Folkman, 1984). 
tico e consequente notificação em virtude de possível constrangimento que a paciente possa experimentar ao falar sobre o assunto devido à vergonha e medos associados (Kachani \& Cordás, 2009; Saunders et al., 2009). Em estudo prospectivo com 227 gestantes usuárias da Maternidade-Escola da Universidade Federal do Rio de Janeiro (UFRJ), onde foi conduzido o presente estudo, observou-se que esta desordem foi referida por $14,4 \%$ das mulheres atendidas no período de $2005-2006$, sendo que $42,1 \%$ praticavam-na diariamente (Saunders et al., 2009).

Por fim, considerando o caráter multifatorial, a dificuldade de determinação das causas da picamalácia e sua associação com desfechos pré-natais indesejáveis, o prognóstico dessa desordem deverá ser tão mais favorável quanto mais precoce for diagnosticada e tratada sob enfoque interdisciplinar, incluindo médicos, psicólogos e nutricionistas, como recomendado por Kachani e Cordás (2009). Diante do exposto e considerando a insignificante produção científica sobre o tema, nosso objetivo foi analisar indicadores emocionais (sinais de estresse, ansiedade e depressão) de enfrentamento (coping) de gestantes com Diabetes Mellitus Gestacional e relatos de prática picamalácia na gestação atual, buscando discutir possíveis relações entre estes indicadores e a prática da picamalácia que possam explicar o desejo pela ingestão de substâncias inadequadas com pequeno ou nenhum valor nutricional durante a gestação, vivenciado por estas mulheres.

\section{Método}

O estudo, que teve delineamento descritivo exploratório foi conduzido com uma amostra de conveniência de 07 gestantes adultas usuárias de serviço de pré-natal referência em Diabetes Mellitus na gestação da Maternidade Escola da Universidade Federal do Rio de Janeiro (UFRJ). Todas foram diagnosticadas com Diabetes Mellitus Gestacional seguindo protocolo assistencial da instituição (Bornia, Costa, \& Amim, 2013; International Association of Diabetes and Pregnancy Study Groups Consensus Panel [IADPSG], 2010), quando eram identificadas com Picamalácia por meio de entrevista padronizada
(Saunders et al., 2009) aplicada nas consultas com o nutricionista e que incluía questões sobre a ocorrência e a frequência do transtorno, além das substâncias ingeridas.

Após, as participantes eram encaminhadas para a pesquisa e agendada avaliação psicológica e entrevista individuais. Nesse momento, as participantes eram esclarecidas sobre os objetivos da pesquisa, quando assinavam o Termo de Livre Consentimento Esclarecido, aprovado por Comitê de Ética da instituição (CAEE n. 06386412.1 .0000 .5275 de 06\09\2013). A coleta de dados ocorreu em sala própria do Setor de Psicologia da instituição, quando eram aplicados os instrumentos de avaliação de estresse, ansiedade, depressão e coping, descritos a seguir.

Para avaliação do estresse foi usado o ISSL, Inventário de Sintomas de Estresse para Adultos (Lipp, 2000), que avaliava sinais e sintomas de estresse em três tipos (somático/físico, psicológico ou mistos) e o nível de estresse nas quatro fases a seguir:

1. Alerta: fase conhecida como reação saudável ao estresse, na qual o indivíduo é capaz de lidar com o estímulo estressor e em seguida retornar ao seu equilíbrio;

2. Resistência: caracterizada pela busca de homeostase do desequilíbrio gerado pela fase anterior, em que o indivíduo, com maior desgaste emocional, fica suscetível a desenvolver algum quadro sintomatológico e manifestações psicossociais;

3. Quase-exaustão: caracterizada pelo enfraquecimento da pessoa e consequente dificuldade de se ajustar e resistir à situação, em que, apesar do grande cansaço, o indivíduo é capaz ainda de executar algumas tarefas; e

4. Exaustão: considerada a fase patológica do estresse, caracterizada pela permanência de estímulos estressantes que desencadeiam falhas na capacidade adaptativa do organismo, com importante desequilíbrio interior, ocorrência de depressão e produtividade nula.

Para avaliar sintomatologia de ansiedade e depressão foram usadas as Escalas BECK, Inventários BAI e BDI, que tem como objetivo avaliar sinais e sintomas de ansiedade e depres- 
são, respectivamente. Com propriedades psicométricas baseadas em normas e padronização brasileira (Cunha, 2001), o BAI e o BDI são escalas de auto-relato compostas por 21 itens contendo afirmações descritivas em que, através da soma de cada resposta, obtém-se um escore total que pode variar de 0 a 63 , de acordo com os seguintes níveis de gravidade crescente de sinais e sintomas:

1. Para ansiedade: (a) mínimo $=01$ a 07 ; (b) leve $=08$ a 15 ; (c) moderada: 16 a 25 ; e (d) grave: 26 a 63; e,

2. Para depressão: (a) mínimo: 01 a 09; (b) leve $=10$ a 16 ; (c) moderado: 17 a 29; e (d) grave: 30 a 63 .

Já, para avaliar o tipo de coping, ou seja, o modo de enfrentamento adotado pelas gestantes para lidar com os estressores durante a gravidez, foi usado a Escala "Modos de Enfrentamento de Problemas" - EMEP (Seidl, Tróccoli, \& Zannon, 2001) solicitando as participantes que respondessem a escala considerando o contexto de sua gravidez de risco com diabetes. A EMEP permite uma análise clínica ou qualitativa das estratégias de enfrentamento utilizadas frente ao estressor em contextos de saúde. Com base na avaliação da resposta a 45 itens, distribuídos em fatores, obtém-se quatro tipos de coping: (a) Estratégias de enfrentamento focalizadas no problema; (b) Estratégias de enfrentamento focalizadas na emoção; (c) Estratégias de enfrentamento focalizadas em práticas religiosas/pensamento fantasioso; e (d) Estratégias de enfrentamento focalizadas na busca de suporte social. Trata-se de uma escala tipo Likert de cinco pontos, variando do "eu nunca faço isso" até "eu faço isso sempre". Para análise da EMEP, realizava-se o somatório dos valores assinalados em que a maior média dos escores indicava a principal estratégia de enfrentamento usada individualmente; além disso, seus autores afirmam que a EMEP permite uma análise do tipo de enfrentamento predominante e possui uma estrutura fatorial coerente $\mathrm{e}$ significativa com base na literatura sobre coping (Seidl et al., 2001).

Cabe esclarecer que o Inventário BDI foi aplicado no dia da entrevista, uma vez que este instrumento foi introduzido posteriormente na coleta de dados com objetivo de analisar mais especificamente relações entre indicadores de depressão e a prática da picamalácia. E, ainda, devido à impossibilidade de agendar a entrevista com uma das gestantes antes dela ter o bebê, apenas seis participantes foram entrevistadas e avaliadas com o Inventário BDI. Para as entrevistas foi usado um roteiro semi-estruturado com nove perguntas, elaborado para a pesquisa com objetivo de coletar o relato verbal das participantes sobre sua vivência relacionada à gravidez de risco com diabetes e sua relação com a prática da picamalácia na gestação. O roteiro teve como propósito compreender como esse transtorno alimentar aparece no período gestacional, assim como o desejo da mulher por consumir combinações de alimentos inusitadas ou substâncias não comestíveis e as possíveis relações com indicadores de estresse, ansiedade, depressão e de coping. As entrevistas foram realizadas individualmente, sendo gravadas e posteriormente transcritas para análise do conteúdo pela metodologia de Bardin (1977/2006).

Dados das entrevistas foram analisados e processados de forma descritiva e qualitativa, com base na análise dos relatos das participantes e na frequência de ocorrência das seguintes macro-categorias: (a) "Motivação para a prática da picamalácia"; (b) "História pessoal da prática da picamalácia"; (c) "Realização do desejo"; (d) "Satisfação na ingestão"; (e) "Compartilhamento do desejo"; (f) "Suporte familiar". Já os dados da avaliação psicológica pelos Inventários BAI, BDI, ISSL e a EMEP foram analisados e processados de acordo com as normas de cada instrumento.

\section{Resultados}

A maioria das gestantes $(61,52 \%)$ possuía ensino médio e $85,8 \%(n=06)$ delas exerciam algum trabalho remunerado (manicure, doméstica, auxiliar de escritório, técnica de enfermagem, entre outros). Maior parte $(85,8 \%)$ das gestantes tinha companheiro $(n=6)$ e filhos $(n=6)$ e, com relação à religião, $85,8 \%$ se declararam cristãs (evangélicas, principalmente) e 14,2\% espiritas, praticantes ou não. 
Dados de ansiedade identificados pelo Inventário BAI das Escalas BECK estão apresentados na Figura 1, em que pode ser observado que nenhuma gestante apresentou sinais de ansiedade em nível severo. Por outro lado, $71,4 \%(N=06)$ foram classificadas com nível moderado de ansiedade; enquanto que $28,6 \%(N$ =02) apresentaram nível leve.

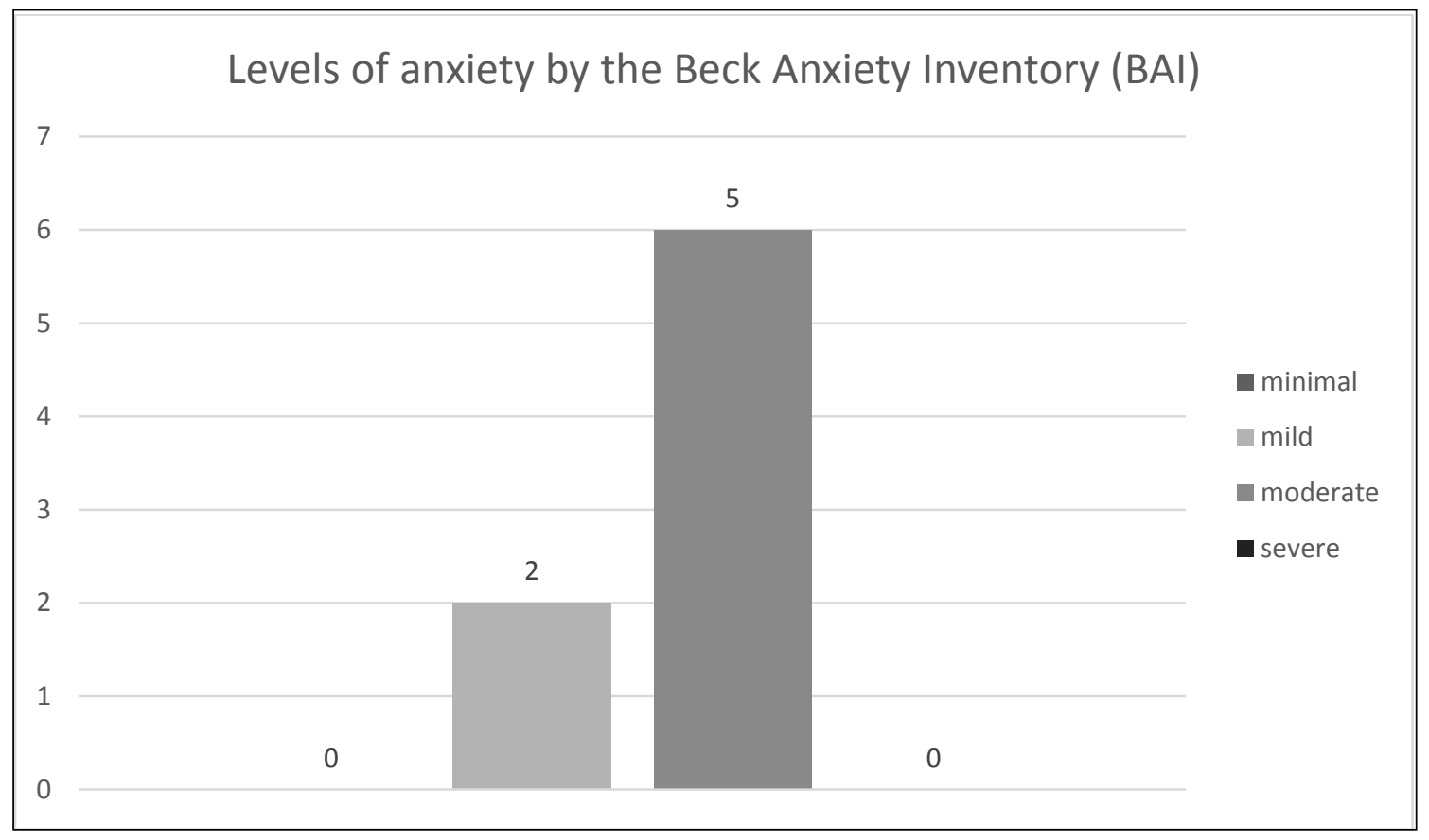

Figura 1. Indicadores de ansiedade pelo Inventário BAI $(\mathbf{N}=07)$.

Em relação à depressão, os dados obtidos pelo Inventário BDI estão apresentados na Figura 2. Todas as gestantes apresentaram algum grau de depressão, sendo quatro gestantes com sinais de depressão moderada $(66,6 \%)$, uma severa $(16,6 \%)$ e apenas uma leve $(16,6 \%)$.

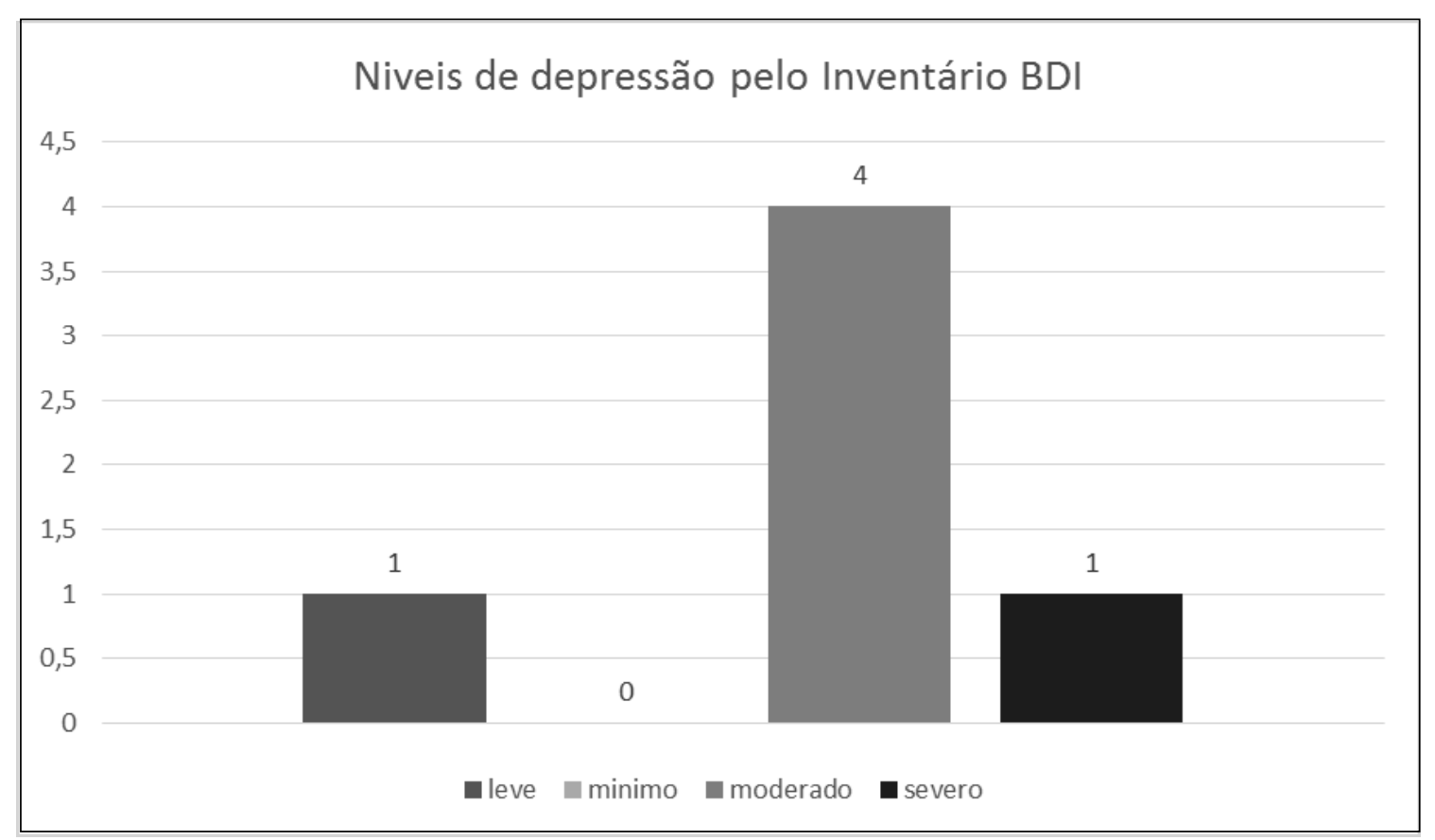

Figura 2. Indicadores de depressão pelo Inventário BDI $(\mathbf{N}=06)$. 
Na Figura 3 são encontrados os resultados da avaliação do estresse pelo Inventário de Sintomas de Estresse para Adultos (ISSL). Pode-se observar que cinco gestantes $(71,4 \%)$ se encontravam na fase de resistência e duas $(28,6 \%)$ na fase de exaustão; enquanto nenhuma gestante apresentou sinais e sintomas de estresse característicos da fase 1 e 3 , alerta e quase-exaustão, respectivamente.

Na Tabela 1 pode ser analisada a prevalência do tipo de enfrentamento usado pelas gestantes identificado pela Escala de Modos de Enfrentamento de Problemas (EMEP).

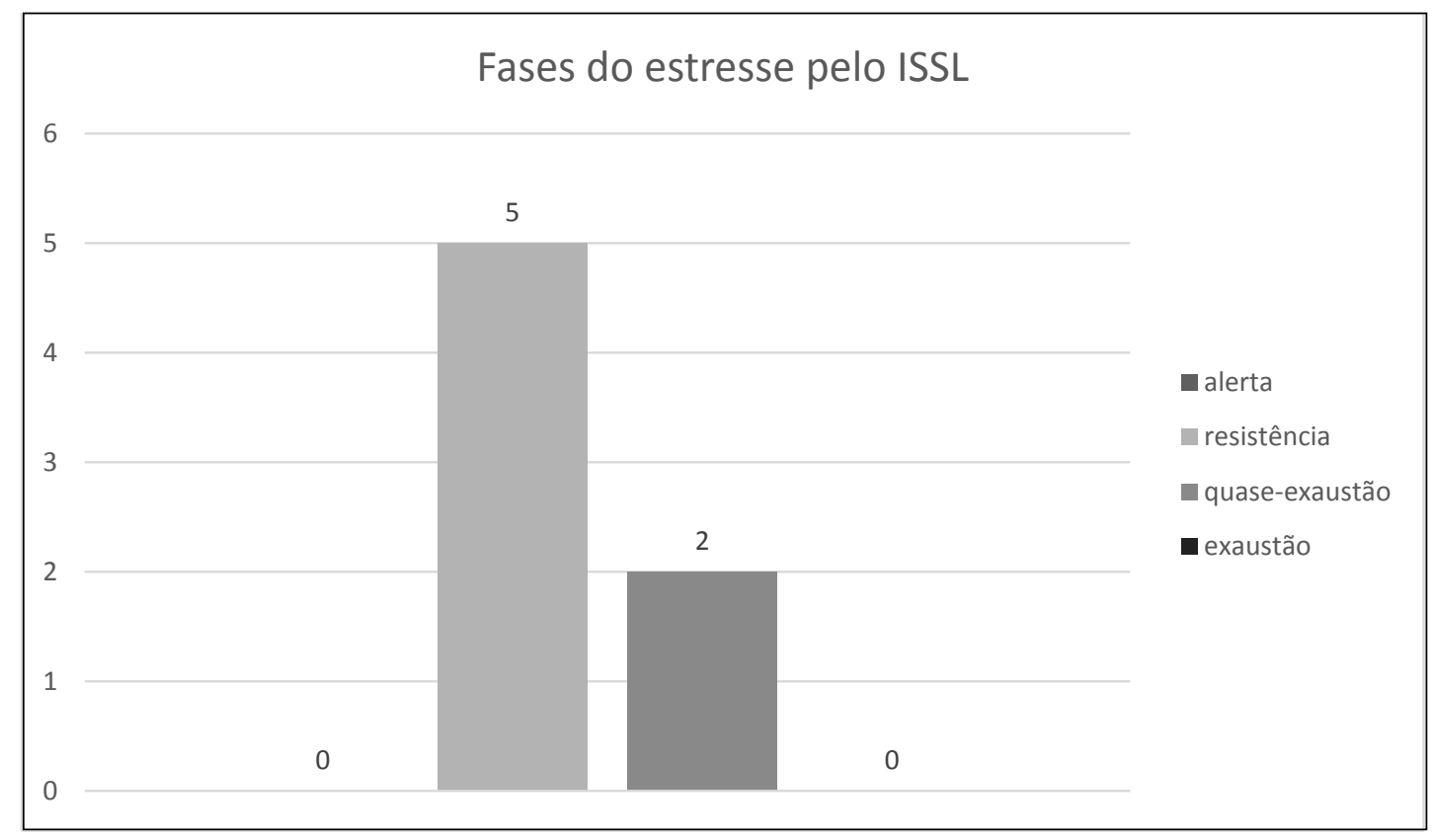

Figura 3. Fases do estresse pelo ISSL, Inventário de Sintomas de Estresse de Lipp (n=07).

\section{Tabela 1}

Frequência das Gestantes por Tipo de Coping Mais Prevalente, de Acordo com a EMEP, Escala de Modos de Enfrentamento de Problemas

\begin{tabular}{ccc}
\hline Classificação EMEP & Frequência & Percentil \\
\hline Coping focado na prática religiosalpensamento fantasioso & 03 & 42,8 \\
Coping focado na busca por suporte social & 03 & 42,8 \\
Coping focado no problema & 01 & 14,4 \\
Coping focado na emoção & 0 & 0 \\
\hline Total & 07 & 100,0 \\
\hline
\end{tabular}

De acordo com avaliação do coping pela EMEP, nenhuma participante utilizou estratégias de enfrentamento focalizadas na emoção. Podemos ainda observar que $42,8 \%$ das gestantes tiveram como estratégia de enfrentamento (coping) mais prevalente a busca por práticas religiosas\pensamento fantasioso. Igualmente, outras três participantes $(42,3 \%)$ utilizaram o coping focado na busca de suporte social, enquanto somente em uma prevaleceu o uso do coping focado no problema.

No relato verbal das gestantes foram identificados alguns temas principais, evidenciados ao longo das entrevistas e desdobrados nas macro-categorias anteriormente citadas, cujos resultados serão apresentados descritivamente. Cabe destacar que, as combinações de alimentos citadas pelas gestantes eram na maioria de 
frutas com sal, como por exemplo, manga verde, abacaxi, cajá e limão com sal, que estão entre as combinações mais citadas pelas participantes e identificadas como prática de picamalácia segundo os critérios diagnósticos estabelecidos. No entanto, houve ainda relatos de gestantes que consumiam combinações exóticas como pé-de-moleque com pão, além de ingerirem substâncias não apropriadas para o consumo, como detergente, naftalina e sabonete Phebo.

Com relação à "Motivação para a prática da picamalácia" foi observado que todas compartilhavam a crença de "saciar um desejo", apesar de relatarem que tinham consciência de que alguns desejos ultrapassavam o recomendável pela dieta, como sugerido nesse relato:

Eu sentei e comi uma bacia cheia, é aquela coisa com vontade. Muito gostoso. . . O da manga e o açúcar faziam eu descuidar da dieta. Porque no da manga eu estava com pressão alta, e o outro eu estava com diabetes e não pode comer o açúcar. Tinha que usar adoçante, mas depois eu parei, não tive mais, para poder controlar a diabetes. (G3) Em alguns casos, este desejo se centrava, inclusive, na ingestão de produtos não propriamente comestíveis e até combinações exóticas e inusitadas, que poderiam comprometer a saúde da gestante e do bebê, como "chupar naftalina".

Ao serem perguntadas sobre a motivação que fomentava o desejo, $75 \%$ citaram o cheiro dos alimentos elou produtos como sendo o provocador desse desejo, como por exemplo, neste relato:

Não podia ir ao banheiro e ver o sabonete que eu queria comer, eu esfregava ele bem na língua mesmo, e depois eu ficava passando mal porque o gosto não era igual o cheiro, e o que me atraía era o cheiro, e você fica salivando, salivando, para você ver que o gosto não é igual o cheiro. (G4)

No momento da entrevista houve uma situação em que uma delas, inclusive, relatou que ao falar da substância sentia até o cheiro da mesma e, por conseguinte, a vontade de ingeri-la: - " $D a ́$ vontade. Igual o cheiro do coco que eu estou sentindo agora. Me lembro logo do detergente, porque ele tem esse cheiro e aí dá vontade de comer, de tomar o detergente" (G2).

Ao longo das entrevistas apareceu o tema "História pessoal da prática da picamalácia", quando as participantes relataram sobre o aparecimento do desejo peculiar pelas combinações exóticas. Frutas com sal foram citadas, por algumas gestantes, como desejos que já faziam parte da sua rotina e que durante a gestação aumentaram a frequência. Uma das gestantes, inclusive, relata que o consumo de limão com sal era compartilhado pela família: "É comum sim. A minha mãe não, mas meu irmão sim. Era coisa de criança. Era assim, a gente comprava para fazer uso do limão, e ai, era só colocar o sal" (G1). Contudo, o desejo pela ingestão de substâncias inadequadas foi citado como um comportamento que iniciou ao longo da gestação. Algumas entrevistadas acrescentaram ainda que desde sua primeira gestação experimentaram desejos exóticos e praticavam a picamalácia. Nos relatos também foi observado que a ingestão de combinações alimentares exóticas e o desejo por substâncias não comestíveis eram percebidos, por estas mulheres, como sensações estranhas, mas ao mesmo tempo com uma grande exigência de satisfação: "Eu fico relutando um pouco, se for uma coisa muito estranha, mas chega uma hora que fica dificil, porque a vontade é muito grande, você saliva muito" (G4). Em sua maioria, as gestantes declararam que o desejo pela ingestão de combinações exóticas elou substancias não comestíveis desapareceram após o fim da gestação.

De acordo com os relatos das gestantes foi possível perceber que, no que se refere a categoria "Realização do desejo", havia prazer envolvido no ato de praticar a picamalácia, ou seja, de se alimentar com substâncias consideradas inapropriadas para ingestão ou combinações extravagantes, como ilustra o relato a seguir: “. . - porque a vontade é muito grande, você saliva muito. Eu estou com muita vontade de comer cajá. Só de falar a minha boca já saliva. Mas tem que ser com sal, porque ele puro não adianta" (G4). A realização do desejo aparece como algo enfático em todas as entrevistas e é reconhecido pelas gestantes como algo que as con- 
some intensamente e que necessita ser satisfeito imediatamente. Dessa forma, as gestantes relatam uma espécie de representação subjetiva para esse desejo, caracterizando-o como uma experiência avassaladora que aparece subitamente e, por vezes, de forma incontrolável, como exemplificado nas falas a seguir: "Esse que é o desejo. Que você come tanto que até extrapola" (G3); "O desejo é súbito e vem a qualquer hora" (G4).

Todas disseram sentir uma vontade incontrolável de realizar o desejo que, enquanto não é satisfeita, controla seus pensamentos e só é coibida quando elas tomam consciência de que pode trazer danos para sua saúde e para a do seu bebê. Cabe destacar o caso de uma gestante cujo desejo era chupar naftalina e que, mesmo após ser alertada pela sua médica acerca dos prejuízos para sua saúde e a do bebê, continuava sentindo o desejo de chupar naftalina, ainda que ciente do perigo por se tratar de substância química. O relato da gestante a seguir demonstra o quão avassalador pode ser o desejo presente na prática da picamalácia, nesse caso unicamente controlado pela consciência dos prejuízos à saúde e à gestação:

Eu procuro me controlar. Eu tentava controlar e controlava na medida do possivel, mas tentava não ficar com aquela vontade. Igual à naftalina, que eu conversei com a doutora e ela falou que fazia mal então eu parei. Me dava vontade mas eu não comia. ... Igual da naftalina, passou não sei quanto tempo, voltou. Eu estava na casa de uma conhecida, eu me controlei para não chupar porque eu senti um cheiro muito forte. . . . Porque eu sei que faz mal. Não é uma coisa que não dá pra controlar. (G2)

As gestantes, em sua maioria, relataram que a experiência de realização do desejo ainda provocava nelas a sensação da "boca cheia d'água", o que expressava o próprio desejo. Neste sentido, a frequência da ingestão desses alimentos variava de acordo com cada gestante, podendo ser semanalmente ou diariamente. Algumas disseram consumir todos os dias $(25 \%)$, outras somente quando estavam em determinados lugares $(50 \%)$, como na casa de familiares ou no trabalho; e outras ainda relataram que somente consumiam quando estavam sozinhas (25\%). O relato de um caso extremo de ter consumido durante três dias somente a combinação exótica de abacaxi com sal foi ainda observado:

Eu comi 3 dias seguidos, só abacaxi com sal, sem comida nenhuma, só abacaxi com sal. Eu até emagreci e a médica brigou. Não tomava café, não comia nem nada, era só abacaxi com sal. Bebi água normal, mas comida não, só tinha vontade de comer abacaxi com sal. (G4).

O local e o modo de consumir as substâncias não comestíveis ou combinações esquisitas foi algo singular para cada gestante. Enquanto uma participante relatou que praticava a pica até no seu local de trabalho, outra relatou que realiza a prática de forma escondida. Essa última quando questionada sobre o porquê deste seu comportamento, respondeu: "Eu escondia, por causa desse problema meu de pressão, por isso que eu não falei pra ninguém". E acrescenta: "esse era o meu segredo" (G3). E, ainda, duas delas relataram que consumiam na frente de seus familiares, mesmo quando eles reclamavam e ressaltavam o fato de fazer mal ao desenvolvimento de sua gestação.

No que se referia ao tema "Satisfação na ingestão", as entrevistadas compartilhavam um sentimento de satisfação após a prática da picamalácia, destacando estar "matando a sua vontade" e gerando "uma sensação de alívio". Contudo, também demonstravam preocupação com a sua saúde e a do seu bebê, chegando algumas declararem se sentir culpadas, como por exemplo: "Gerava uma culpa, porque eu sabia que ai o exame ia dar alterado" (G1), fazendo referência ao consumo excessivo de açúcar; além daquelas que, assim, ficavam preocupadas com as consequências da prática da pica: "Depois... eu tinha medo da pressão. Então dava uma preocupação. E às vezes quando dava a vontade de comer eu falava: 'Não posso, não posso'. Porque comer isso vai afetar a minha saúde e a saúde dela" (G3)

$\mathrm{Na}$ análise da categoria "Compartilhamento do desejo", um fator interessante apareceu no discurso de algumas gestantes. Ficou evidente nos relatos das gestantes a não existência 
do sentimento de vergonha ao falar sobre os seus desejos, sobre as combinações inusitadas que ingeriam e até mesmo sobre a ingestão de substâncias não comestíveis. As entrevistadas relataram um estranhamento em relação a esses tipos de desejo, mas que não resultava necessariamente em uma limitação em falar sobre o assunto. Inclusive buscavam compartilhar essa vivência com outras mulheres grávidas a fim de encontrar semelhanças nos comportamentos e hábitos alimentares e, assim, se identificar positiva ou negativamente com seus pares, como no exemplo:

Uma conhecida minha que está grávida, que é esposa do primo do meu esposo. Ela tá grávida e é diabética também. Mas ela está viciada. E eu conheci ela na casa da minha sogra, onde eu estava comendo, ai ela viu e pediu para eu pegar pra ela também a manga verde. (G2)

Por fim, o tema do "Suporte familiar" se apresentou como um elemento importante na implicação da gestante em relação à saúde da díade mãe-bebê. A presença dos familiares junto a gestante neste período parecia garantir a esta mulher uma percepção de que ela não estava sozinha ou desamparada, mas sim que estava sendo cuidada, mesmo nos momentos em que esses familiares faziam um papel coibidor do seu desejo, quer seja a mãe da gestante:

A primeira vez eu chupei 5 limões, porque no dia seguinte tinha que fazer exame. A minha mãe brigou e eu não fiz mais e a minha boca começou a estourar aqui, começou abrir machucado, então eu parei porque estava ardendo muito. $(\mathrm{G} 2)$

Ou até o companheiro: ". . . e o meu marido falou: 'Pelo amor de Deus S., o desinfetante não!' Se eu passar em algum lugar e sentir o cheiro eu quero tomar" (G4, fazendo referência ao desejo de consumir desinfetante).

\section{Discussão}

Gestantes que praticam a picamalácia apresentam sinais significativos de estresse em algum nível (Ayeta et al., 2015; Woods, Melville, Guo, Fan, \& Gavin, 2010), assim como um número significativo de mulheres desencadeiam ou pioram sintomas de ansiedade (Vythilingum, 2008). Variáveis psicológicas, como ansiedade e depressão, podem afetar a mãe no período pré-natal, contribuindo para desfechos obstétricos indesejáveis, como a prematuridade, o baixo peso e problemas de desenvolvimento infantil (Ding et al., 2014; Van Batenburg-Eddes et al., 2013). Associado a estes indicadores emocionais, o estresse materno pode se relacionar também a desfechos negativos (Davis \& Sandman, 2010; Rodrigues \& Schiavo, 2011; Woods et al., 2010). Tais condições emocionais têm maiores índices na gravidez de risco (Ding et al., 2014), quando estão presentes condições que potencializam o estresse e condições emocionais negativas, como ansiedade e depressão, sendo importante investigar a relação destes riscos com o enfrentamento dos mesmos.

Relacionando os fatores de risco, DMG e Picamalácia, com os aspectos psicológicos estudados, ansiedade, estresse e depressão, podemos supor a partir desses resultados preliminares que ambos apontam para riscos à saúde mental na gestação. Assim como na pouca literatura sobre o assunto (Araújo et al., 2013; Ayeta et el., 2014; Morrison et al., 2014; Nicklas et al., 2013; Nolan et al., 2011; Silva et al., 2004), nossos resultados sugerem que o diagnóstico de DMG é um fator gerador de ansiedade, depressão e estresse em gestantes, sendo necessário à adoção de estratégias de enfrentamento (coping) resilientes a fim de prevenir que a gestante desenvolva um transtorno alimentar, como a Picamalácia, como uma forma de se proteger da vivência de sofrimento psíquico característico da DMG.

Conforme estudo anterior (Ayeta et al., 2015), foi observado prevalência de dois tipos de enfrentamento, o coping focado no suporte social e o coping focado em práticas religiosas $\backslash$ pensamento fantasioso nas gestantes com relato de picamalácia. Quanto ao coping focado na busca por suporte social podemos perceber nas entrevistas que o apoio da família foi muito importante para a maioria dessas mulheres, quer seja por compartilharem as mesmas preferências alimentares, quer seja pelos familiares impedirem a realização do seu desejo incontrolável por 
substâncias, por vezes, tóxicas (detergentes, por exemplo) ou exóticas (manga com sal, limão com sal, entre outros). De fato, o suporte social tem uma função importante quando se trata de uma gravidez de risco, quando a família passa a ser a principal rede de apoio/suporte para a mulher que vivencia intercorrências gestacionais. Nesse contexto de gravidez, a família, em geral, exerce papel importante favorecendo uma evolução satisfatória dessa gestação a partir da adaptação dos familiares às recomendações médicas, com adesão ao tratamento da patologia presente na gestante (Petroni, Silva, Santos, Marcon, \& Mathias, 2012).

O segundo modo de enfrentamento que prevalece em gestantes com relatos de picamalácia é o coping focado em práticas religiosas mento fantasioso (Ayeta et al., 2015). Tal tipo de coping também foi percebido nos discursos daquelas mulheres, quando relatavam o receio de acarretar consequências "desastrosas" para o bebê como motivação para não realizar o desejo de praticar a picamalácia, como ilustra o seguinte relato de uma gestante sobre a gravidez de sua mãe e seu nascimento:

. . Mas eu acabei nascendo de cabelo arrepiado, porque ela queria comer um frango com quiabo e a minha avó não fez e eu acabei nascendo assim. Você tinha que ver as minhas fotos, de quando eu era pequena de cabelo arrepiado. Que ridículo! Eu falei: "porque não rasparam a cabeça dessa criança".

Crenças e superstições, representadas por pensamentos fantasiosos característicos do $\mathrm{Co}$ ping focado na busca de práticas religiosas pensamento fantasioso tal como ilustra o relato acima, são exemplos que explicam esse tipo de coping. Além disso, é comum na gestação que a mulher experimente ao longo de toda a gravidez experiências psicológicas, fisiológicas e sociais únicas, provocando a construção de expectativas diversas em relação ao bebê que resultam em um processo de idealização da maternidade. Particularmente as fantasias angustiantes sobre a integridade do feto (Soulé, 1992) podem transformar a gravidez em uma vivência psicológica particularmente estressante e propensa a crises
(Sarmento \& Setúbal, 2003), quando a gestante pode recorrer a um tipo de enfrentamento como o coping focado no pensamento fantasioso, a exemplo do que ilustra a fala de outra entrevistada, que ingeriu tijolo ao longo da gestação:

... Um colega meu falou que isso é psicológico, que tem alguma coisa que está faltando para o bebê, que manda uma mensagem para o meu cérebro em forma de tijolo, ai eu falei, que é uma casa em construção.

Concomitante a isso não podemos esquecer de que a literatura tem enfatizado aspectos culturais da prática da picamalácia na gestação, quando a mulher grávida se baseia na crença de que deve ingerir tal substância para que seu bebê nasça sadio e perfeito (Fiestas-Teque, 2014; Young, 2010).

Somado a isso, é importante considerar possíveis relações entre os fatores de risco estudados, DMG e Picamalácia, no enfrentamento do estresse e ansiedade já característico da gravidez. Com relação ao enfrentamento (coping) também foi observado na análise das entrevistas que as mulheres atribuíam vários sentidos ao seu desejo avassalador (grifo dos autores) de ingerir alimentos incomuns ou substâncias tóxicas. Caberia, inclusive, a interpretação de que tal atitude mais poderia parecer uma tentativa de se desculpar do seu ato incontrolável que as arrebatava de forma súbita e quase irresistível, como no caso da gestante que consumia naftalina.

Se por um lado, as mulheres tinham esclarecimento sobre as consequências imediatas e diretas para sua gestação desse comportamento alimentar inapropriado, adquiridos nas consultas com o médico e com o nutricionista; por outro, elas contavam com uma tradição familiar de ingerir combinações incomuns ou mesmo alimentos sem valor nutricional, como o consumo de limão com sal que perpetuava um hábito familiar, possivelmente justificado por forte crença transgeracional (Ahmed et al., 2012; Kachani \& Cordás, 2009; Thihalolipavan et al., 2013; Young, 2010). Isto parece justificar a prática da picamalácia naquelas gestantes, já presente desde gestações anteriores. Tal como observado no relato das entrevistadas sobre sua história de pratica da picamalácia, quando o 
desejo e a prática em si já faziam parte da rotina, apenas aumentando a frequência durante aquela gestação.

A presença da sintomatologia de ansiedade, depressão e estresse pode estar relacionada à picamalácia como uma forma de alívio do estresse e sintomas depressivos provenientes da situação de risco materno e fetal decorrente da DMG, como sugere a literatura na área (Ayeta et al., 2015; Kachani \& Cordás, 2009; Young, 2010). No estudo, todas as gestantes apresentaram algum tipo de indicador emocional em níveis diversos e importantes de serem discutidos. Todas apresentaram sinais de estresse e ansiedade, com sinais importantes que equivalem dizer que as mulheres estavam em uma fase caracterizada pela busca de homeostase do desequilíbrio gerado por uma fase de estresse anterior que as deixaram em estado de alerta e quando ela experimenta maior desgaste emocional e fica suscetível a desenvolver algum quadro sintomatológico e manifestações psicossociais (Lipp, 2000).

A presença do estresse relacionado aos sinais de ansiedade aponta para a importância de estudar a picamalácia como um "mediador do estresse" (Ayeta et al., 2015; Young, 2010), sobretudo se considerarmos a gravidez como momento de repercussões emocionais significativas para a mulher (Sarmento \& Setúbal, 2003). Podemos afirmar que, não olvidando as limitações para generalização dos dados, sinais de estresse e ansiedade são comuns em casos de picamalácia, já confirmado por estudo anterior (Ayeta et al., 2015). Nesses casos, podemos supor que essas manifestações psicossomáticas podem ser entendidas como um ato sintoma que procura descarregar, o mais rapidamente possível, toda a tensão criada por acontecimentos internos ou externos que demandam da pessoa se reequilibrar psiquicamente diante das exigências psicológicas da gravidade de uma situação, como é a DMG. Tal tensão, como nos alerta McDougall (2003), pode se relacionar tanto a estados afetivos penosos quanto a estados afetivos agradáveis, que podem provocar grande excitação e euforia, mas que são percebidos e interpretados pela pessoa como proibidos ou perigosos para ele. Esta hipótese poderia ainda explicar a prática da picamalácia para aquelas mulheres que relataram experimentar grande satisfação e prazer na realização do desejo pela prática, mas que, de forma ambivalente, experimentavam culpa e preocupação por estar prejudicando sua saúde e do seu bebê.

Como uma resposta de proteção ao estresse psicológico, a picamalácia pode estar relacionada à ansiedade presente desde o primeiro trimestre de gravidez devido a necessidade de maior controle da alimentação. Tal controle pode ser fator gerador de estresse para a gestante e sua família, resultando em irritabilidade já decorrente das disfunções hormonais próprias da gestação (Vieira \& Parizoto, 2013). Cabe destacar que foi observado nas entrevistas relatos de mulheres que declararam ter interrompido o consumo de combinações estranhas de alimentos ou mesmo substâncias tóxicas ou não alimentícias depois do segundo trimestre. Isto sugere que a picamalácia, de fato, pode se apresentar como uma forma de alívio da sintomatologia ansiedade e do estresse (Ayeta et al., 2015; Kachani \& Cordás, 2009; Young, 2010) presente particularmente no início da gravidez, quando são maiores as mudanças e as exigências de restrição alimentar, própria da gravidez e com destaque especial na gestação com diabetes.

Em resposta ao objetivo de buscar mais especificamente relações entre sintomatologia depressiva e a prática da picamalácia, chama atenção o fato de que todas as gestantes apresentaram algum grau de depressão, sendo a maioria de sinais de depressão moderada e até depressão severa para uma gestante. Tais dados devem ser discutidos com cuidado, uma vez que a depressão é um transtorno importante, com repercussões até o puerpério de desfechos para depressão pós-parto e psicose puerperal. Podemos supor que esses sinais de depressão seriam indicadores importantes a serem mais amplamente investigados à luz da experiência individual de cada mulher. Nessa direção, não podemos deixar de considerar que cada mulher vivencia de maneira única as mudanças e exigências presentes em cada gestação, o que justifica que a prática da picamalácia ocorra em uma gravidez e não necessariamente em outra. Dessa forma, é fundamental entendermos a história particular de cada 
mulher como expressão de um discurso concernente a um contexto específico de gravidez, em que mudanças físicas e psicológicas e seus efeitos simbólicos poderão resultar em medos, angústias, ansiedades, depressões, mal-estar e, por vezes, somatizações daquilo que não foi possível ser expressado em palavras.

Por outro lado, em análise intragrupo foi possível observar que as gestantes que apresentaram sinais moderados de depressão também apresentavam alguma doença crônica além do diabetes, como hipertensão arterial por exemplo. Essa condição crônica caracterizaria um fator de risco psicológico a mais de impacto na saúde mental desta mulher. Segundo Quevedo et al. (2006), a grávida identificada como alto risco apresenta dificuldades para as adaptações emocionais exigidas pelo novo papel que lhe é exigido, o de mãe, acrescendo-se nessa situação a demanda de várias outras emoções. Além disso, em decorrência do fator de risco, surge o medo real em relação a si própria (o que está ocorrendo com seu corpo) e ao seu filho (o temor de anormalidades), sendo necessária atenção especial à condição de vulnerabilidade emocional destas gestantes, que pode indicar e/ou potencializar um estado depressivo, a exemplo do que encontramos em nossa pesquisa.

Importante ainda ressaltar que na gestante com sinais severos de depressão foi possível observar que, além da presença de doença crônica, ocorreu o estresse em nível de exaustão com o coping focado no problema. Esse quadro reflete uma concomitância de fatores de risco para a saúde da gestante e seu bebê e sinalizam uma possível emergência de um estado depressivo. Destaca-se como fundamental uma atenção preventiva a situações de potencial gravidade como essa, ainda que o tipo de enfrentamento adotado por essa gestante seja o coping focado no problema, que representaria um enfrentamento resolutivo e mais adaptativo frente ao estresse. No entanto, estratégias de coping focado no problema por si só não garantiriam um enfrentamento resiliente da situação que prevenisse a ocorrência de uma depressão, já que esse tipo de coping tende a ser mais utilizado nas situações avaliadas como modificáveis, o que não era o caso. Para aquela mulher o risco gestacional acompanharia todo o percurso da gravidez, não sendo possível uma reversão da situação, mas apenas o manejo das circunstâncias agravantes da condição clinica existente. Por outro lado, pode-se supor também que adotar um enfrentamento focado no problema permitiria que a mulher adotasse estratégias de racionalização que as auxiliaria na busca de informações e alternativas para lidar com o problema (Lazarus \& Folkman, 1984), se engajando, assim, em mecanismos resolutivos para enfrentar sua condição de vulnerabilidade evitando riscos maiores que estivessem relacionados ao aparecimento de quadro depressivo, por exemplo.

Estudo de Saunders et al. (2009) concluiu que a picamalácia pode ser um comportamento de adaptação, de aceitação, de representação de uma necessidade fisiológica ou de um desejo da gestante por uma substancia não nutritiva, fazendo referência a este período gestacional que convida a mulher a se readaptar diante das transformações vivenciadas. Igualmente, nossos dados nos convidam a compreender que na vivência da gestação existem fatores psicológicos que podem explicar o desejo daquelas mulheres pelo consumo de combinações atípicas ou substâncias inadequadas com pequeno ou nenhum valor nutricional: a picamalácia. $\mathrm{O}$ fato das mulheres que praticavam a picamalácia encontrarem semelhanças entre seus comportamentos e hábitos alimentares com os de outras mulheres grávidas, nos faz refletir sobre como a condição de gravidez, proporciona a mulher uma aceitação de desejos que são socialmente considerados inapropriados e agressores a saúde.

Diferente da literatura (Kachani \& Cordás, 2009), não foi comum dentre as participantes a vergonha em compartilhar a pratica da picamalácia com seus pares e familiares. No entanto, relato como o da gestante que praticava a picamalácia de forma escondida nos permite pensar e refletir sobre o quão significativo é a satisfação do desejo para aquelas mulheres. Tal desejo pode, inclusive, representar um lugar de empoderamento para elas por proporcionarem a si mesmas algo que lhes cause prazer, alivio, satisfação e outros adjetivos utilizados por elas 
próprias, como: "Parece que eu fico fixada naquilo... Sei lá! Uma coisa estranha" (G2, fazendo referência ao desejo) e "Matei a vontade $e$ foi bom porque eu não coloquei pra fora" (G3, fazendo referência a sensação após a ingestão de manga verde com sal). Em muitos casos, apesar do mesmo desejo que provocava contentamento também resultava em culpa, repudia e preocupação, as participantes continuavam realizando a prática da picamalácia, negando a ambivalência de sentimentos vivenciada por elas, principalmente quando se tratava de substancias não comestíveis, como sabonete, detergente, desinfetante, tijolo etc., como no exemplo: "Quando são essas coisas estranhas sim. É um pouco anti-higiênico né?" (G4, fazendo referência ao consumo do sabonete Phebo).

Parte das entrevistadas relatou dificuldades em realizar a prática da picamalácia na frente de seus familiares. Logo, o suporte familiar pode ter sido facilitador para o enfrentamento das adversidades provenientes desse momento de suas vidas, ainda mais considerando que todas as entrevistadas tinham diabetes e apresentaram sintomatologia de estresse, ansiedade e depressão consideráveis. Assim, a percepção do suporte familiar naquele momento pode ter auxiliado também a conter a prática da picamalácia devido ao auxílio oferecido pelos familiares que, por vezes, representavam a interdição que elas precisavam para frear o ímpeto de realização do desejo presente na prática da picamalácia. Ainda assim, houveram casos em que as gestantes relataram que, por mais que os familiares não apoiassem a ingestão de certos conteúdos alimentícios ou não, estavam por perto doando sua escuta, sua companhia e seu auxilio, como no exemplo: "Muito. Tudo o que acontece na minha gestação, não só nessa, mas em todas, ele está presente. Ele chega e pergunta, quer saber como está o neném. $A$ vontade dele era estar aqui comigo agora.

." (G4, fazendo referência à participação do companheiro na gestação).

Relatos sobre a presença do suporte familiar reforçam a ideia de que o enfrentamento de gestantes com picamalácia é focado na busca de suporte social (Ayeta et al., 2015). Os níveis mínimos de ansiedade apresentados por estas ges- tantes ajudam a confirmar que o suporte familiar exerce influência no manejo do período gestacional, tal como observado nas entrevistas das participantes deste estudo. Um adequado suporte social proporciona apoio em momentos particularmente importantes e críticos ao longo da vida, como os períodos de parto, pós-parto e puerpério, quando é exigido da mulher um papel proativo desde o nascimento até os cuidados posteriores ao recém-nascido. Assim, o suporte social favorece para as gestantes e puérperas maior controle do ambiente e autonomia, fornecendo esperança, apoio e proteção (Coutinho, Baptista, \& Morais, 2002). Além disso, este suporte é fundamental para o bem-estar de mulheres grávidas, já que, segundo Petroni et al. (2012), a família, por exemplo, interfere de forma positiva diminuindo a ansiedade e transmitindo confiança no caso da gestação de alto risco. Logo, o suporte social, assim como o suporte familiar, são indicadores importantes na manutenção da saúde mental e no enfrentamento de situações vulneráveis.

Práticas de picamalácia desde a primeira gestação, como repetição de um comportamento, pode ser fator que corrobora para perpetuação do ato de consumir substâncias exóticas, por ser algo conhecido e de fácil manipulação. E, ainda, a repetição da picamalácia pode até mesmo estar relacionada a dificuldades de se controlar diante de um desejo mais intenso ou mesmo uma compulsão, uma vez que estudos indicam que tal compulsão, assim como a inibição do comportamento instintivo em que se baseia a prática da picamalácia, podem estar relacionados ao sistema de receptação de serotonina (Bhatia \& Kaur, 2014). Essa hipótese poderia ser testada em futuros estudos em que se investigasse o processo de enfrentamento relacionado ao sistema de autorregulação das emoções e comportamento, o que não foi exatamente o foco desse estudo, mas coloca-se como sugestão para novas pesquisas.

Por fim, cabe enfatizar que a discussão dos dados do nosso estudo apresenta limitações, uma vez que o tamanho da amostra restringe a generalização dos resultados. Limitações amostrais impediram realizar uma análise quantitativa correlacional dos dados, o que impossibilita conclusões mais amplas e robustas. No entanto, acredi- 
ta-se que devido a pouca literatura cientifica na área, reflexo da própria dificuldade de acessar a população diagnosticada com picamalácia (Ayeta et al., 2015; Kachani \& Cordás, 2009; Saunders et al., 2009), nossos achados podem contribuir para uma discussão inicial com base no objetivo que nos propusemos, quer seja discutir possíveis relações entre indicadores emocionais e de enfrentamento, a picamalácia e o desejo que permeia esta prática na gestação de risco com Diabetes Mellitus Gestacional.

\section{Conclusão}

Por fim, cabe concluir que as escolhas alimentares não devem ser entendidas, somente, segundo a racionalidade técnico-científica, mas, também, como formas explicativas e singulares de cada indivíduo elou grupo (Baião \& Deslandes, 2010). Nesse sentido, podemos pensar a prática da Picamalácia como formas de enfrentamento dessas mulheres frente às inquietudes e incertezas vivenciadas ao longo da gestação, um sentido subjetivo que transcende o sentido médico nutricional focalizado no hábito alimentar. Nessa direção, nossos dados nos confrontam com a necessidade de atenção à saúde mental desse grupo em especial, com efetivos serviços terapêuticos que incluam, além de um diagnóstico clinico diferencial, ações terapêuticas eficazes para minimizar o impacto dos riscos inerentes à Síndrome da Picamalácia, associada ou não às condições crônicas de saúde durante a gravidez como, por exemplo, a DMG. Nessas ações, destaca-se a participação do psicólogo, quer seja no atendimento direto a paciente e sua família, quer seja na interconsulta aos demais profissionais de saúde, que devem ser sensibilizados para prestar atenção e assistência nesses casos, identificando-os e proporcionando a gestante uma assistência integral.

Contudo, seriam necessários maiores estudos, sobretudo com caráter longitudinal, cujo objetivo seja articular os fatores de risco que envolvem o período gestacional, a condição de saúde crônica que a gestante apresenta, a emergência de comportamentos peculiares e a relação entre a Síndrome da Picamalácia e os indicadores emo- cionais (depressão, ansiedade, estresse) a fim de compreendermos mais amplamente essa realidade vivenciada pela gestante e pensarmos ações preventivas para a saúde da díade mãe-bebê. Futuras investigações sobre a vivência feminina desse e outros problemas, também no período de pós-parto, possibilitariam ampliar a discussão sobre os problemas psicológicos presentes com frequência no puerpério, como a depressão pós-parto por exemplo. Muitas vezes sub-diagnosticada, a depressão faz parte das psicopatologias comuns deste período, sobretudo em mulheres com perturbações do comportamento alimentar, podendo se relacionar diretamente com o modo como a mulher enfrenta o estresse típico da chegada do bebê e a vivência da sua maternagem.

\section{Referências}

Ahmed, S., Abdullahi, H., \& Adam. I. (2012). Practice of pica among pregnant women in Khartoum, Sudan. International Journal of Gynecology and Obstetrics 118, 71-76.

American Psychiatric Association. (2013). Diagnostic and statistical manual of mental disorders ( $5^{\text {th }}$ ed.). Arlington, VA: Author.

Araújo, M. F. M., Pessoa, S. M. F., Damasceno, M. M. C., \& Zanetti, M. L. (2013). Diabetes gestacional na perspectiva de mulheres gravidas hospitalizadas. Revista Brasileira de Enfermagem, 66(2), 222-227.

Ayeta, A. C. C., Cunha, A. C. B., Heidelmann, S. P., \& Saunders C. (2015). Fatores nutricionais e psicológicos associados com a ocorrência de picamalácia em gestantes. Revista Brasileira de Ginecologia e Obstetrícia, 37, 571-577.

Baião, M. R., \& Deslandes, S. F (2010). Práticas alimentares na gravidez: Um estudo com gestantes e puérperas de um complexo de favelas do Rio de Janeiro (RJ, Brasil). Ciência Saúde Coletiva, 15(Supl. 2), 3199-3206. doi:10.1590/S141381232010000800025

Bardin, L. (2006). Análise de conteúdo (L. de A. Rego \& A. Pinheiro, Trads.). Lisboa: Edições 70. (Original publicado em 1977)

Bhatia, M. S., \& Kaur, N. (2014). Pagophagia - A common but rarely reported form of pica. Journal of Clinical \& Diagnostic Research, 8(1), 195-196. 
Bornia, R. G., Costa, I. B., Jr., \& Amim, J., Jr. (2013). Protocolos Assistenciais. Maternidade Escola. Universidade Federal do Rio de Janeiro. Anestesiologia, Neonatologia, Obstetrícia. Recuperado em http://www.me.ufrj.br/portal/images/ stories/pdfs/obstetricia/diabetes_mellitus.pdf

Coutinho, D. S., Baptista, M. N., \& Morais, P. R. (2002). Depressão pós-parto: Prevalência e correlação com o suporte social. Infanto - Revista de Neuropsiquiatria da Infância e Adolescência, 10, 63-71.

Cunha, J. A. (2001). Manual da versão em Português das Escalas Beck (J. A. Cunha, Trad.). São Paulo, SP: Casa do Psicólogo.

Davis, E. P., \& Sandman, C. A. (2010). The timing of prenatal exposure to maternal cortisol and psychosocial stress is associated with human infant cognitive development. Child Development, 81(1), 131-148.

Ding, X., Wu, Y., Xu, S., Zhu, R., Jia, X., Zhang, S., ...Tao, F. (2014). Maternal anxiety during pregnancy and adverse birth outcomes: A systematic review and meta-analysis of prospective cohort studies. Journal of Affective Disorders, 159, 103-110.

Dunker, K. L. L., Alvarenga, M. S., \& Alves, V. P. O. (2009). Transtornos alimentares e gestação Uma revisão. Jornal Brasileiro de Psiquiatria, 58(1), 60-68.

Erdem, G., Hernandez, X., Kyono, M., Chan-Nishina, C., \& Iwaishi, L. (2004). In-utero lead exposure after maternal ingestion of Mexican pottery: Inadequacy of the lead exposure questionnaire. Clinical Pediatrics, 43, 185.

Ezzeddin, N., Zavoshy, R., Noroozi, M., Jahanihashemi, H., \& Riseh, S. H. (2015). Prevalence and risk factors for pica during pregnancy in Tehran, Iran. Eating and Weight Disorders, 20, 457-463.

Fiestas-Teque, L. (2014). Pica en anemia severa: a propósito de un caso. Revista de NeuroPsiquiatría, 77(2), 123-125.

Gangopadhyay, R., Karoshi. M., \& Keith. L. (2011). Anemia and pregnancy: A link to maternal chronic diseases. International Journal of Gynecology \& Obstetrics, 115(1), 11-15.

International Association of Diabetes and Pregnancy Study Groups Consensus Panel. (2010). International association of diabetes and pregnancy study groups recommendations on the diagnosis and classification of hyperglycemia in pregnancy. Diabetes Care, 33(3), 676-682.

Kachani, A., \& Cordás, T. A. (2009). Da ópera-bufa ao caos nosológico: Pica. Revista de Psiquiatria Clinica, 36(4), 162-169.

Lazarus, R. S., \& Folkman, S. (1984). Stress, appraisal and coping. New York: Springer.

Lipp, M. E. N. (2000). Manual do inventário de sintomas de stress para adultos de Lipp (ISSL). São Paulo, SP: Casa do Psicólogo.

Mcdougall, J. (2003). Théatre du corps. Paris: Folio essais.

Mikkelsen, T. B., Andersen, A. N., \& Olsen, S. F. (2006). Pica in pregnancy in a privileged population: Myth or reality. Acta Obstetricia et Gynecologica, 85, 1265-1266.

Morrison, M. K., Lowe, J. M., \& Collins, C. E. (2014). Australian women's experiences of living with gestational diabetes. Women and Birth, 27, 52-57.

Nicklas, J. M., Miller, L. J., Zera, C. A., Davis, R. B., Levkoff, S. E., \& Seely, E. W. (2013). Factors associated with depressive symptoms in the early postpartum period among women with recent gestational diabetes mellitus. Maternal and Child Health Journal, 17, 1665-1672.

Nolan, J. A., McCrone, S., \& Chertok, I. R. (2011). The maternal experience of having diabetes in pregnancy. Journal of the American Academy of Nurse Practitioners, 23(11), 611-618.

Petroni, L. M., Silva, T. C., Santos, A. L., Marcon, S. S., \& Mathias, T. A. F. (2012). Convivendo com a gestante de alto risco: A percepção do familiar. Ciência, Cuidado e Saúde, 11(3), 535-541.

Quevedo, M. P., Lopes, C. M. C., \& Lefevre, F. (2006). Os significados da maternidade para mulheres cardiopatas e diabéticas com gravidez de risco. Revista Brasileira Crescimento Desenvolvimento Humano, 16(1), 12-21.

Rodrigues, O. M. P. R., \& Schiavo, R. A. (2011). Stress na gestação e no puerpério: Uma correlação com a depressão pós-parto. Revista Brasileira de Ginecologia e Obstetrícia, 33(9), 252-257.

Sarmento, R., \& Setúbal, M. R. V. (2003). Abordagem psicológica em obstetrícia: Aspectos emocionais da gravidez, parto e puerpério. Revista Ciências Médicas, 12(3), 261-268. 
Saunders, C., Padilha, P. C., Líbera, B. D., Nogueira, J. L., Oliveira. L. M., \& Astulla, A. (2009). Picamalácia: Epidemiologia e associação com complicações da gravidez. Revista Brasileira Ginecologia Obstetrícia, 31(9), 440-446.

Seidl, E. M. F., Tróccoli, B. T., \& Zannon, C. M. L. C. (2001). Análise fatorial de uma medida de estratégias de enfrentamento. Psicologia Teoria e Pesquisa, 17(3), 225-234.

Silva, L., Santos, R. C., \& Parada, C. M. G. L. (2004). Compreendendo o significado da gestação para grávidas diabéticas. Revista Latino-Americana de Enfermagem, 12(6), 899-904.

Simpson, E., Mull, J. D., Longley, E., \& East, J. (2000). Pica during pregnancy in low-income women born in Mexico. The Western Journal of Medicine, 173, 20-40.

Sociedade Brasileira de Diabetes. (2015). Diretrizes da Sociedade Brasileira de Diabetes. São Paulo, SP: Autor.

Sociedade Brasileira de Endocrinologia e Metabologia. (2008). Diabetes mellitus gestacional. Revista da Associação Médica Brasileira, 54(6), 477-480.

Soulé, M. (1992). Le psychiatre dans un service de medicine fetale et diagnostic anténatal. In M. Soulé, F. Begoin-Guignard, H. Cohen, F. Daffos, D. David, P. Faure, ...B. Schaal (Eds.), Introduction à la psychiatrie fetale. Les aspects psychologiques et psycopathologiques en medicine fetale et diagnostic prénatal. Paris: ESF.
Thihalolipavan, S., Candalla, B. M., \& Ehrlich, J. (2013). Examining Pica in NYC pregnant women with elevated blood lead levels. Maternal and Child Health Journal, 17, 49-55.

Van Batenburg-Eddes, T., Brion, M. J., Henrichs, J., Jaddoe, V. W. V., Hofman, A., Verhulst, F. C., ...Tiemeier, H. (2013). Parental depressive and anxiety symptoms during pregnancy and attention problems in children: A cross-cohort consistency study. Journal of Child Psychology and Psychiatry, 54(5), 591-600.

Vieira, B., \& Parizotto, A. (2013). Alterações psicológicas decorrentes do período gravídico. Unoesc \& Ciência - ACBS, 4(1), 79-90.

Vythilingum, B. (2008). Anxiety disorders in pregnancy. Current Psychiatry Reports, 10(4), 331-335.

Woods, S. M., Melville, J. L., Guo, Y., Fan, M. Y., \& Gavin, A. (2010). Psychosocial stress during pregnancy. American Journal of Obstetrics \& Gynecoly, 202(1), 61, e1-7. 7.

Young, S. L. (2010). Pica in pregnancy: New ideas about an old condition. Annu. Revista de Nutrição, 30, 403-422. 